\title{
Maternal health care in Nigeria: Do community factors moderate the effects of individual-level Education and Ethnic origin?
}

\author{
Dorothy N Ononokpono \\ Department of Sociology and Anthropology University of Uyo \\ P.O.Box 1017, Uyo Nigeria \\ doraono@gmail.com
}

\begin{abstract}
Maternal mortality ratio in Nigeria is still high despite the initiation of the safe motherhood initiative. The poor maternal health situation has been attributed to inadequate use of maternal health care services. This study examines whether community factors moderated the association between individual factors and the use of skilled ANC. Data on 17560 women aged 15-49 years drawn from 2008 Nigeria Demographic and Health Survey were used. Multilevel logistic regression models were used for analysis. Results reveal variations in the use of skilled ANC across the Northern and Southern regions. Residence in communities with a high proportion of women that delivered in a health facility increased the odds of skilled ANC utilization. Community education and poverty moderated the association between individual factors and the utilization of skilled ANC. To improve the use of ANC and increase the pace towards achieving the sustainable Development Goal-improved maternal health in the post 2015 era, increasing health facility delivery, women's education and targeting poverty alleviation programs in disadvantaged communities should be taken into consideration.
\end{abstract}

Keywords: Antenatal care, community, maternal health care, multilevel models, Nigeria

\section{Résumé}

Ratio de mortalité maternelle au Nigéria est encore élevé malgré l'ouverture de l'initiative pour une maternité sans risque. La mauvaise santé maternelle situation a été attribuée à l'insuffisance des services de soins de santé maternelle. Cette étude examine si facteurs communautaires a modéré l'association entre facteurs individuels et l'utilisation de l'ANC qualifiés. Données sur 17560 femmes âgées de 15 à 49 ans tirées de 2008 le Nigeria enquête sur la démographie et la santé ont été utilisés. Multiniveau modèles de régression logistique ont été utilisées pour l'analyse. Les résultats révèlent des variations dans l'utilisation de I'ANC qualifiés partout dans les régions du Nord et du Sud. Résidence dans des communautés avec une forte proportion de femmes que livré dans un établissement de santé a accru les chances de l'ANC qualifiés l'utilisation. L'éducation communautaire et la pauvreté modérée l'association entre facteurs individuels et I'utilisation de I'ANC qualifiés. Pour améliorer l'utilisation de I'ANC et de l'augmentation du rythme vers la réalisation de l'objectif de développement durable et l'amélioration de la santé maternelle dans le poste 2015 ère, à accroître la santé installation de livraison, l'éducation des femmes et le ciblage des programmes de lutte contre la pauvreté dans les communautés défavorisées devraient être prises en considération.

Mots clés : soins prénatals, de la communauté, les soins de santé maternelle, modèles à plusieurs niveaux, le Nigéria

\section{Introduction}

Globally, 210 maternal deaths per 100,000 live births occur due to pregnancy related complications (WHO, 20I4). Although, maternal mortality ratio has declined in developed countries, the rate remains high in developing countries. Sub-Saharan Africa accounts for 62 percent of world's maternal deaths (WHO 20I4). With as estimated maternal mortality ratio of 560 per 100,000 live births (World Bank, 20I4), Nigeria ranks second in the list of countries with the highest rate of maternal mortality in the world. The importance of the use of maternal health care in reducing maternal mortality is well recognized and some researchers have argued that health care services are important in determining the overall 
maternal mortality levels in developing countries (lkamari 2004; Onah, 2006).

Appropriate maternal health care is important in the management of complications of pregnancy (Magadi et al. 2003). Antenatal care service is one of the major interventions recommended for the reduction of maternal and newborn deaths (Titaley et al. 2010). It also provides some preventive interventions for pregnant women. These include tetanus immunization, nutrition education, and counselling regarding their plans for delivery and postpartum family planning (Ikamari, 2004). Antenatal care ensures optimal outcome for both mother and baby, and is important in monitoring pregnancy and reduction of morbidity and mortality (NPC \& ICF Macro, 2009). World Health Organization (WHO) recommended antenatal care from a skilled health worker (example midwife, doctor or nurse). Furthermore, antenatal care provides opportunity for pregnant women to identify complications associated with pregnancy and benefit from other interventions including counselling on healthy lifestyles and management of complications (Kistiana, 2009).

Despite the benefits of antenatal care, the Nigeria Demographic and Health Survey (NDHS) 2008 report indicated that less than two third (58 percent) of women in Nigeria received skilled antenatal care during pregnancy (NPC \& ICF Macro, 2009).

The poor maternal health situation in Sub-Saharan African countries and Nigeria in particular is worrisome; and attempts have been made by researchers to understand the determinants of this phenomenon. While most studies on maternal health care have focused on the influence of individual-level determinants, the influence of community-level factors has received less attention. As a result of the emphasis on the influence of individual-level factors, efforts to improve the use of maternal health care services have concentrated on addressing individuallevel characteristics such as maternal education, ethnic origin and household wealth status which have been identified as important determinants of maternal health care. Studies have shown that knowledge about the individual-level factors is not sufficient to address the problem of poor maternal health care behaviour (Mackian, 2003; Rahman, 2000). Hence, it is important to look beyond the individual-level characteristics. The rationale for considering the characteristics of the community in which people live has been well recognized (Robert \& House, 2000; Blalock, 1984). For instance, individuals live in communities and their health care behaviour could be influenced by the characteristics of other members of the communities in which they reside.

Although some studies have examined the independent effects of community factors on the use of maternal health care services (Babalola and Fatusi, 2009; Stephenson et al 2006), there is a dearth of studies on the moderating effects of community factors on the association between individual-level factors and maternal health outcomes. Extant studies in Nigeria have shown that maternal education and ethnic origin are strong determinants of maternal health care behaviour (Doctor 20II; Wall, 1998). Furthermore, other studies have established that many contextual or aggregate variables such as community education and poverty which have been hypothesized to influence health vary over geographic and social units (Boco, 20l0; Vu, 2005).

Most communities in Sub-Saharan African countries have different characteristics and vary in terms of socio-economic development and maternal health outcomes. Nigeria is ethnically and socially diverse, and there are variations in health outcomes across the different communities in the country. Hence it is important to examine whether the influence of selected individual-level factors (education and ethnic origin) on maternal health care could be mitigated by community factors. This will help to identify individual and community factors that could be targeted for interventions to improve maternal health.

\section{Literature Review and Theoretical framework}

A number of factors have been associated with the use of maternal health care. Some studies in Asia found that women's autonomy was strongly associated with antenatal care utilization (Bloom et al., 200 I; Shaikh \& Hatcher 2004). The authors noted that men control all aspects of resources and decide when and where women should seek health care. In addition, Matthews and Gubhaju (2004) observed that women who hold strong views on the right to refuse sex with their partner were more likely to use antenatal care; married women were more likely to use antenatal care if they make more decisions in the household, if they were educated, if they have educated partner and if they regularly listened to the radio or watch television.

Cultural beliefs and practices were important determinants of maternal health-seeking behaviour (Shaikh et al. 2008; Griffiths \& Stephenson, 200I). This indicates that the use of maternal health care is not only related to the individual's choice, but also to a large extent depends on the socio-cultural arrangements of communities. Socio-cultural factors impinge negatively on women's access to health care. In Islamic countries for instance, a woman's place is thought to be restricted to the home and she has few options for economic independence (Ojanuga and Gilbert, 1992). In addition, the cultural institution of purdah segregates Muslim women from public places 
and greatly impacts on their health care-seeking behaviour.

Social support from family members was significantly associated with the use of antenatal care. Stressing the role of mothers-in-law in decisions to seek ANC, Simkhada et al. (2010) noted that mothers-in-law sometimes have a positive influence, especially when encouraging women to seek antenatal care, but more often it is negative. The main reasons why mothers-in-law do not support/encourage antenatal check-ups include expectations regarding pregnant women fulfilling their household duties, perceptions that antenatal care was not suitable, as well as strained power relations between mothers-in-law and daughters-inlaw.

Maternal education was significantly associated with the use of antenatal care. Education in particular has been identified as relevant in the enhancement of female autonomy in terms of decision making regarding their health. An educated woman with higher socio-economic status has the ability to make appropriate decisions concerning the use of maternal health care. (Abadine et al, 2008). Trinh et al (2007) identified external environment, predisposing characteristics and need as the most related factors to using antenatal care. Acceptability of the provider services which depends on the satisfaction of service is a major determinant of the utilization of antenatal care (Dolla, 2008).

Wealth, parity and age affect a woman's chances of having optimal antenatal care (Mbuagbaw \& Gofin, 20I I). For instance, poor wealth status and previous maternity experiences of high parity women may present difficulties in the utilization of skilled antenatal care. In addition, younger women tend to be in a high risk group, and thus more likely to use skilled ANC. Other studies in Nigeria found that ethnicity (Babalola \& Fatusi, 2009; Ononokpono et al., 20l3), income yielding occupations (Oguntunde et al., 2010), gestational age at initial antenatal care booking (Galadanci et al., 2007), and quality of antenatal care (Awusi et al. 2009) were strongly associated with the utilization of ANC.

Community level factors such as availability of subcentre in the primary sampling unit and distance to the nearest primary health centre were significantly associated with the utilization of antenatal care in Uttar Pradesh (Tiwari, 2010). Women were more likely to use health care services if the health care centers were available and in close proximity to where they reside. Other studies found that Community poverty and community education were strong determinants of maternal health care. For instance, communities with a high concentration of poor households and a low concentration of welleducated residents are not likely to have the resources necessary to develop, sustain and access high quality health care services (Gage, 2007).

As noted earlier, most previous studies on maternal health care have focused on the individual and familial factors and neglected the moderating effects of community characteristics. An examination of the moderating effects of community factors would help in understanding whether the magnitude of the effects of the individual-level factors will change as a function of some community factors.

From the theoretical perspective, social ecological theory of health promotion developed by Stokols (1996) provided an understanding of how community conditions are related to health behaviour. Social ecological framework emphasized multiple effects and the interrelations between environmental conditions and human behaviour and wellbeing. The theory stresses that environmental settings have multiple, physical, social and cultural dimensions that can influence health outcomes or health behaviour. These include various geographic, architectural, technologic, organizational and sociocultural conditions that are present within a particular community or cluster, which are linked to health outcomes at both individual and community levels. The social conditions such as the level of education in the community, poverty, proportion of health facility delivery and mass media exposure in the community as well as, physical conditions including urban-rural residence and region of residence could influence the use of antenatal care. Furthermore, cultural conditions such as ethnic origin and religion are important determinants of maternal health care. The ecological framework also emphasized the influence of intrapersonal factors such as maternal education, age and household wealth status on health care behaviour.

The literature has established that both individual and community factors such as community poverty, community education, ethnic origin and maternal education were important determinants of health outcomes (Boco 2010, Gage 2007; Uthman 2010). Motivated by the recent interest in the effects of these factors on health outcomes, this study seeks to investigate whether community factors could moderate the effects of the individual-level factors on maternal health care. Specifically, the objective of this study is to examine whether community factors moderate the association between individual-level education and ethnic origin on the use of skilled antenatal care in Nigeria. We hypothesize that: the magnitude of the effects of education and ethnic origin on the use of skilled ANC will change as a function of community-level factors. 


\section{Data and Methods}

The data analyzed in this study was drawn from the 2008 Nigeria Demographic and Health Survey (NDHS), a cross-sectional survey designed to provide information on population and health indicators at the national and state levels. The sample frame for the survey was lists of EAs developed from the 2006 population census. The primary sampling unit (PSU), which is referred to as the cluster was selected from the lists of Enumeration Areas (EAs). Sample for the survey was selected using a stratified two-stage cluster design, made up of 888 clusters; 286 in the urban and 602 in the rural areas. Of the 888 clusters, data were collected in 886 PSUs. This was because two PSUs could not be accessed due to disturbances in the areas. In all, a total sample of 33,385 women aged 15-49 years and 15,486 men aged 15-59 were selected in the survey. Details of the methods used in the NDHS have been published somewhere (NPC and ICF Macro, 2009). The sample included in the analysis reported in this study was limited to 17560 women who had their most recent delivery in the five years before the survey.

The outcome variable is antenatal care. This is a dichotomous variable coded I if a woman received ANC from a skilled health provider (doctor, nurse or midwife) and 0 otherwise. The explanatory variables were grouped into individual- and community-level factors. These variables were selected because the literature shows that they were significantly associated with the use of maternal health care. The individual-level factors include maternal age at last birth, education, religion, ethnic origin, occupation, women's autonomy, and household wealth index. Maternal age was calculated by subtracting the century month code (CMC) of the date of birth of the child from the date of birth reported by the mother and categorized into three groups: 15-24, 2534 and 35-49. Maternal educational attainment was categorized into three: no education, primary, and secondary or higher. Religion was classified as Muslim, Christian and Traditional religion/Others, while ethnic affiliation was categorized as Hausa/Fulani/Kanuri, Igbo, Yoruba and Others. Occupation was defined as the type of work respondents do. This was re-grouped and categorized as formal employment $\mathrm{n}$ (professional/technical/managerial/clerical/sales/skille d manual workers), agricultural employment, unskilled manual workers and unemployed. Woman's autonomy is measured as decision making on a woman's own health care. Household wealth index is the DHS wealth index and was determined through Principal Component Analysis (from Factor Analysis) and based on household assets (e.g. type of flooring, water supply, electricity, radio, television, refrigerator and type of vehicle). This was divided into five quintiles and each quintile represents a relative measure of a household's socioeconomic status (Rutstein and Johnson, 2004).

The community level variables examined include place of residence (urban and rural), region of residence, community hospital delivery, community women's education, community mass media exposure, and community poverty. Region of residence was categorized as: North Central, North East, North West, South East, South-South and South West. Community hospital delivery is the proportion of delivery in a health facility in the primary sampling unit (PSU). This measure was divided into three tertiles and categorized as low, medium and high. Community women's education is the proportion of women with secondary and higher in the PSU. The measure was divided into three tertiles and categorized as low, medium and high. Community mass media exposure is the proportion of women exposed to mass media in the PSU. The proportion was divided into three tertiles and categorized as low, medium and high. Community poverty was measured as the proportion of women from the poor and poorest wealth quintiles and the measure was divided into three tertiles and categorized as low, medium and high. The measure was divided into three tertiles and categorized as low, medium and high. The community variables were constructed by aggregating the average scores of individual and household characteristics (educational attainment, place of delivery, mass media exposure and household wealth index) to the community (primary sampling units). These measures were then divided into tertiles, and categorized as Low (lowest tertile), medium (middle tertile) and high (highest tertile). A total of 886 PSUs were represented in this study and each cluster was made up of a minimum of 80 households.

Two levels of analyses were done. First descriptive analysis was done using frequency distributions and cross tabulations; and the statistical significance of the variables were determined by the chi square test of association. Second, a multilevel model was estimated to assess the relationship between the measured individual and community level factors and the outcome variable. Multicollinearity of the variables was tested using variance inflation factor (VIF). The VIF was I.5, (that is approximately 2). Stata II.I software was used for all analyses. Multilevel analysis was considered appropriate in order to account for the hierarchical nature of the DHS data (Antai, 2009). A two-level multilevel logistic regression model was estimated at individual-level (level I) and community-level (level 2).

According to $\mathrm{Vu}$ (2005), a two-level multilevel model for a dichotomous outcome uses a binomial 
sampling and a logit link. In level I model, the outcome variable $Y_{i j}$ for individual $i$ living in community $j$ is written as follows:

Probability $\left(Y_{i j}=I \mid B\right)=\Phi_{i j}$

Level I variance $=\left[\Phi_{\mathrm{ij}}\left(\mathbf{I}-\Phi_{\mathrm{ij}}\right]^{*}\right.$

Predicted log odds $\eta_{i j}=\log \left[\Phi_{i j} /\left(I-\Phi_{i j}\right)\right]$

$$
\eta_{q=1}=\beta_{0 j}+\Sigma \beta_{q j} X_{q i j}
$$

\section{Where}

Фijis the probability that the ith individual in the jth community take value "I" ("I" indicates that the event will occur)

$\beta_{0 j}$ is the level I intercept

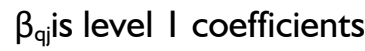

$\mathrm{X}_{\mathrm{qij}}$ is level I predictor $\mathrm{q}$ for ith individual within $\mathrm{jth}$ community

In level 2 model for a two-level model for dichotomous outcome, each of the level I coefficients, $\beta$ qj defined in the level I model becomes an outcome and can be expressed as follows:

$$
\begin{aligned}
& \beta_{q j} \\
& \gamma_{q} S_{q} W_{s_{q j}}
\end{aligned}
$$

$$
=\quad \begin{aligned}
& \mathbf{S}_{\mathbf{q}} \\
& V_{q 0}+\sum \gamma_{q} s W_{s j}+u_{q j}
\end{aligned}
$$

$\mathbf{s = 1}$

Where

$\gamma_{\mathrm{qs}}(\mathrm{q}=0, \mathrm{I}$ $S_{q}$ ) are level 2 coefficients

Wsj are level 2 predictors and $\mathrm{u}_{\mathrm{qj}}$ is level 2 random effects.

Five models were estimated for the outcome variable. The first model is an empty or null model and does not contain explanatory variables but decomposes the total variance into individual and community components. The second model contained individual-level characteristics to enable the assessment of the effects of the individual-level variables on skilled ANC. The third model contained only the community characteristics and this allowed the assessment of the relation of the community variables to the outcome variable. The fourth model is the full model and contained explanatory variables at both the individual and community levels. This allows the assessment of net effects of individual and community variables on the use of skilled ANC.
Model 5 (Final Model) contained the cross-level interactions between some community variables and maternal education and ethnic origin. This allowed us to estimate whether the magnitude of the effect of maternal education and ethnic origin will change as a function of some community factors (community poverty and community maternal education). These variables were selected based on their strong association with maternal health care indicators in previous studies (Babalola and Fatusi, 2009; Gage 2007).

Odds ratio (OR) and $95 \%$ confidence interval, were expressed as fixed effects, while the random effects were expressed as variance partition coefficient (VPC) and proportional change in variance (PCV). The VPC in each Model was calculated based on the linear threshold model method (Merlo et al., 2006). In this case, the individual level variance $\sigma 2 \mathrm{e}$ is equal to $\pi 2 / 3$ (i.e. 3.29). AIC (Akaike information criterion) was used to test the goodness of fit of the models.

\section{Results}

Overall, $45 \%$ of the sample belonged to age group (25-34) (Table I). About (45\%) had no education,

\begin{tabular}{|c|c|c|c|c|}
\hline \multirow[t]{2}{*}{ Characteristics } & \multicolumn{2}{|c|}{ All women } & \multicolumn{2}{|l|}{$\begin{array}{l}\text { Skilled } \\
\text { ANC } \\
\text { (Provider) }\end{array}$} \\
\hline & $\%$ & $\mathbf{n}$ & $\begin{array}{l}\text { Received } \\
\%\end{array}$ & $\begin{array}{c}\text { p- } \\
\text { value }\end{array}$ \\
\hline Maternal age at birth & & & & $<0.001$ \\
\hline $15-24$ & 36.9 & 6476 & 51.9 & \\
\hline $25-34$ & 44.7 & 7847 & 63.9 & \\
\hline $35-49$ & 18.4 & 3238 & 55.7 & \\
\hline Educational & & & & $<0.001$ \\
\hline attainment & 45.4 & 7969 & 31.0 & \\
\hline No education & 22.8 & 4004 & 69.1 & \\
\hline Primary & 25.9 & 4542 & 86.3 & \\
\hline Secondary & 5.9 & 1045 & 97.8 & \\
\hline Higher & & & & \\
\hline Occupation & & & & $<0.001$ \\
\hline Unemployed & 30.4 & 5312 & 47.7 & \\
\hline Formal employment & 41.4 & 7235 & 67.2 & \\
\hline Agricultural & 17.2 & 3005 & 53.5 & \\
\hline employment & 10.9 & 1910 & 58.2 & \\
\hline Manual workers & & & & \\
\hline Religion & & & & $<0.001$ \\
\hline Muslims & 54.3 & 9482 & 42.6 & \\
\hline Christians & 44.0 & 7685 & 77.9 & \\
\hline Traditional/Others & 1.7 & 297 & 38.5 & \\
\hline Ethnic origin & & & & $<0.001$ \\
\hline Hausa/Fulani/Kanuri & 39.6 & 6924 & 31.8 & \\
\hline Igbo & 11.6 & 2033 & 88.3 & \\
\hline Yoruba & 15.0 & 2627 & 88.1 & \\
\hline Others & 33.7 & 5887 & 65.0 & \\
\hline
\end{tabular}
while the lowest proportion had higher education.

Table I Percentage distribution of women by background characteristics and skilled ANC, Nigeria 2008 DHS 


\begin{tabular}{|c|c|c|c|c|}
\hline \multicolumn{4}{|l|}{$\begin{array}{l}\text { Women's autonomy } \\
\text { (decisions over own }\end{array}$} & $<0.001$ \\
\hline health) & 8.8 & 1450 & 73.8 & \\
\hline Wife alone & 33.1 & 5477 & 72.4 & \\
\hline Wife/husband & 58.2 & 9634 & 46.7 & \\
\hline \multicolumn{4}{|l|}{ Husband alone/Others } & $<0.001$ \\
\hline index & 23.1 & 4059 & 23.6 & \\
\hline Poorest & 22.2 & 3898 & 39.9 & \\
\hline Poor & 19.0 & 3332 & 64.3 & \\
\hline Middle & 18.2 & 3187 & 82.4 & \\
\hline Rich & 17.6 & 3084 & 94.1 & \\
\hline \multicolumn{5}{|l|}{ Richest } \\
\hline $\begin{array}{l}\text { Type of place of } \\
\text { residence }\end{array}$ & 30.2 & 5308 & 84.2 & $<0.001$ \\
\hline Urban & 69.8 & 12253 & 46.6 & \\
\hline \multicolumn{5}{|l|}{ Rural } \\
\hline Region of residence & & & & $<0.001$ \\
\hline North Central & 14.3 & 2516 & 65.3 & \\
\hline North East & 15.6 & 2745 & 43.1 & \\
\hline North West & 30.4 & 5337 & 31.3 & \\
\hline South East & 9.1 & 1599 & 87.2 & \\
\hline South South & 13.1 & 2303 & 70.0 & \\
\hline South West & 17.4 & 3061 & 87.5 & \\
\hline $\begin{array}{l}\text { Community women's } \\
\text { education }\end{array}$ & & & & $<0.001$ \\
\hline Low & 42.6 & 7487 & 29.1 & \\
\hline Medium & 29.0 & 5097 & 72.2 & \\
\hline High & 28.3 & 4976 & 86.8 & \\
\hline Community hospital & & & & $<0.001$ \\
\hline delivery & 40.3 & 7072 & 25.2 & \\
\hline Low & 27.4 & 4807 & 70.9 & \\
\hline Medium & 32.4 & 5682 & 87.8 & \\
\hline \multicolumn{5}{|l|}{ High } \\
\hline Community poverty & & & & $<0.001$ \\
\hline Low & 30.5 & 5356 & 90.4 & \\
\hline Medium & 29.4 & 5166 & 62.2 & \\
\hline High & 40.1 & 7039 & 30.2 & \\
\hline $\begin{array}{l}\text { Community mass } \\
\text { media exposure }\end{array}$ & & & & $<0.001$ \\
\hline Low & 32.9 & 5779 & 34.4 & \\
\hline Medium & 32.4 & 5683 & 60.3 & \\
\hline High & 34.7 & 6098 & 78.2 & \\
\hline
\end{tabular}

More than half of the entire sample were Muslims and $44 \%$ were Christians. With respect to occupation, $41.4 \%$ of the sample population were in formal employment, $30.4 \%$ were unemployed, $17.2 \%$ were in the agricultural sector while $10.9 \%$ were manual workers. Ethnic origin of women reflected the dominance of Hausa ethnic group (40\%). About 12\% of the women were Igbo, 15\% were Yoruba and $33 \%$ belonged to the minority ethnic groups. Almost two third of the sample population reported that their husbands or other people have a final say on their utilization of health care. About $45 \%$ of the women were from the two poorest wealth quintiles, while the lowest proportion were from the richest wealth quintile.

Majority of the sample population (70\%) lived rural areas. Also, one third of the women resided in North West, while the lowest proportion lived in South East. Over a third of the study sample lived in communities with a low proportion of educated women, and a low proportion who delivered in a health facility. About $40 \%$ of the women lived in high poverty communities, whereas over a third lived in communities with a high proportion of women who were exposed to mass media.

The bivariate results in Table I show that all the explanatory variables were significantly associated with the use of skilled antenatal care $(p<0.00 \mathrm{I})$. Younger women (25-34 years), those who had secondary/higher education, women who were in formal employment, Christian women, and women who belonged to rich households had a higher frequency of ANC utilization. The proportion of skilled ANC utilization was highest among women who reported that they make decisions alone (73.8\%) and jointly with their husbands $(72.4 \%)$ on their own health care. The proportion of women from lgbo, Yoruba and other minority ethnic groups who used skilled ANC was higher than women from Hausa/Fulani/Kanuri ethnic group. Women who lived in urban areas had a higher proportion (84.2\%) of skilled ANC utilization than rural women. The frequency of the use of skilled ANC was lowest for women from North West (3I.3\%) and North East (43.1\%). The proportion of skilled ANC utilization was higher for women who lived in communities with a high proportion of educated women, a high proportion who had health facility delivery, and a high proportion who were exposed to mass media than women who resided in disadvantaged communities. Conversely, the lowest proportion of antenatal care utilization was found among women who lived in communities with a high proportion of women from poor households.

\section{Multilevel analysis}

Results in model 0 (null Model) (Table 2) indicate a significant variation in antenatal care across communities $(\tau=6.290, p=0.00 \mathrm{I})$. The intracommunity correlation coefficient (ICC) of $65.6 \%$ was related to skilled ANC at the community level. Model I shows the effects of the individual covariates. Only the individual variables were included in this model to examine the independent effects of the individual covariates on skilled ANC. Results reveal that maternal age at last delivery and a woman's autonomy were not significantly related to the use of skilled antenatal care. Women with primary $(O R=2.07, p<00 \mathrm{I})$ and secondary/higher $(O R=3.76, p<0.00 I)$ education were more likely to use skilled ANC compared to women who had no schooling. Traditional religious women had a lower likelihood of skilled ANC utilization. Women of lgbo $(\mathrm{OR}=7.02, \mathrm{p}<0.00 \mathrm{I})$ Yoruba $(\mathrm{OR}=4.46, \mathrm{p}<0.00 \mathrm{I})$ and other minority ethnic origin $(O R=2.56$, $\mathrm{p}<0.00 \mathrm{I})$ had higher odds of skilled antenatal care utilization than Hausa/Fulani/Kanuri women. 
Similarly, women who worked in the formal sector exhibited a higher likelihood of using skilled antenatal care compared to women that were unemployed. As expected, the household wealth index was significantly associated with antenatal care. The odds of ANC utilization was higher for women from richest households $(O R=10.11, p<0.001)$ compared to women from poorest households.

The variance in model I was significant $(\tau=$ 2.095; $\mathrm{p}<0.00 \mathrm{I})$. The intra-community correlation decreased to $38.9 \%$ and the proportional change in variance of receiving skilled antenatal care across communities was $67 \%$. The addition of the individual level variables helped to reduce the community variance from 6.290 to $2.095 \quad(p<0.00 \mathrm{I})$. This indicates that the individual factors were important in explaining the variability in the use of skilled ANC between communities.

Model 2 contained only the community-level variables to enable the assessment of the independent effects of the community-level covariates. Results show that living in North- East $(\mathrm{OR}=0.62, \quad \mathrm{p}<0.00 \mathrm{I}), \quad$ North-West 
Table 2 Multilevel logistic regression odds ratio of the effects of individual and community factors on antenatal care from skilled health provider, Nigeria $2008 \mathrm{DHS}$

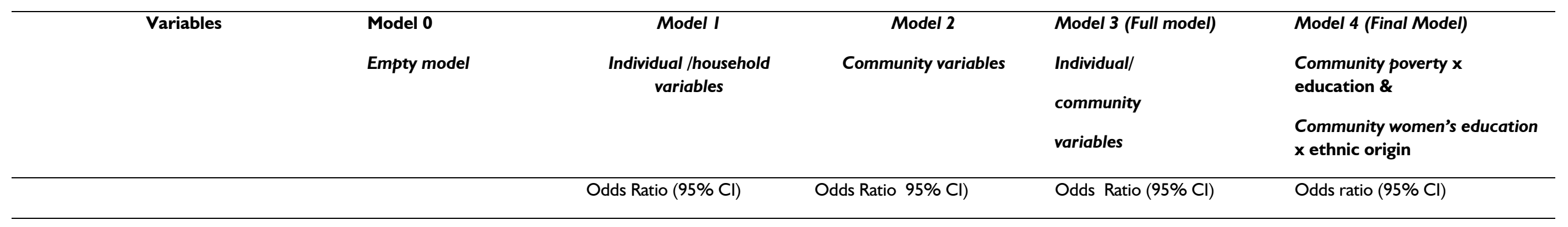

\section{Fixed effects}

Individual characteristics

Maternal age at last birth

$15-24 \quad 1.00$

$25-34$

$1.04(0.94-1.16)$

1.00

1.00

$35-49$

$0.96(0.84-1.09)$

$1.03(0.92-1.14)$

$1.01(0.91-1.11)$

Educational attainment

No education

1.00

$0.93(0.82-1.07)$

0.89 (0.78-I.0I)

Primary

$2.07 * * * *(1.82-2.35)$

1.00

Secondary/Higher

3.76 *** (3.18-4.45)

$1.99 * * * *(1.61-2.46)$

3.64 **** $(2.60-5.10)$

Religion

Muslims

1.00

Christians

$0.98(0.81-1.21)$

$0.53^{* * * *}(0.37-0.77)$

1.00

1.00

0.89 (0.72-I.12)

$1.03(0.84-1.27)$

Traditional/Others

$0.56 * *(0.39-0.82)$ 
African Population Studies Vol. 29, No. I, 2015

\begin{tabular}{|c|c|c|c|c|}
\hline Ethnic Origin & & - & & \\
\hline Hausa & 1.00 & & 1.00 & \\
\hline lgbo & $7.02 * * * *(4.84-10.19)$ & & $3.38 * * * *(1.88-6.08)$ & \\
\hline Yoruba & 4.46 **** $(3.26-6.10)$ & & I.73** $\quad(1.17-2.55)$ & \\
\hline Others & 2.56 **** $(2.09-3.15)$ & & I.98**** (1.5I-2.59) & \\
\hline \multicolumn{5}{|l|}{ Occupation } \\
\hline Unemployed & 1.00 & & 1.00 & 1.00 \\
\hline Formal employment & I.37***** (I.2I-I.55) & - & $1.36 * * *(1.18-1.56)$ & $1.34 * * *(1.22-1.56)$ \\
\hline Agric employment & $0.99(0.85-1.17)$ & & $1.01 \quad(0.86-1.19)$ & $(0.83-1.16)$ \\
\hline Unskilled manual workers & $1.48 * * * *(1.23-1.78)$ & & I.53**** (I.25-1.89) & $1.61 * * *(1.33-1.93)$ \\
\hline \multicolumn{5}{|l|}{ Women's autonomy } \\
\hline Wife alone & 1.00 & - & 1.00 & 1.00 \\
\hline Wife/Husband & $1.4 \mathrm{I}(1.16-1.7 \mathrm{I})$ & & $1.44 * * * *(1.17-1.78)$ & $1.42 * * *(1.17-1.72)$ \\
\hline Husband alone/Others & $1.09(0.91-1.33)$ & & $1.17 \quad(0.96-1.43)$ & $(0.95-1.39)$ \\
\hline \multicolumn{5}{|l|}{ Household wealth index } \\
\hline Poorest & 1.00 & & 1.00 & 1.00 \\
\hline Poorer & I.44**** (I.26-I.66) & & I.34*** $(1.14-1.57)$ & $1.32 * * *(1.16-1.52)$ \\
\hline Middle & $2.55 * * * *(2.17-3.01)$ & - & $1.98 * * * *(1.56-2.5 \mathrm{I})$ & $1.98 * * * *(1.67-2.34)$ \\
\hline Richer & $4.32 * * * *(3.54-5.28)$ & & $2.73 * * *(1.98-3.76)$ & $2.84 * * *(2.3 \mathrm{I}-3.5 \mathrm{I})$ \\
\hline Richest & $10.11 * * * *(7.59-13.47)$ & & $5.24 * * *(3.29-8.34)$ & $5.45 * * *(4.02-7.4 I)$ \\
\hline \multicolumn{5}{|l|}{ Place of residence } \\
\hline Urban & & 1.00 & 1.00 & - \\
\hline Rural & & $0.80(0.60-1.07)$ & $0.82(0.61-1.10)$ & \\
\hline
\end{tabular}


African Population Studies Vol. 29, No. I, 2015

\section{Region of residence}

South West

North Central

North East

North West

South East

South South

Community women's education

Low

Medium

High

Community hospital delivery

Low

Medium

High

\section{Community poverty}

Low

Medium

High

Community mass media exposure

Low

Medium

High

\section{Community poverty $\mathbf{x}$ education}

Low poverty $\mathrm{x}$ none/primary

\subsection{0}

$0.62 * *$ (0.58-1.23)

$0.27^{* * * *}(0.34-0.80)$

I.72** (0.13-0.38)

$0.46 * * * *(0.93-2.28)$

I. $17(0.25-0.6 \mathrm{I})$

1.00

$2.37 * * * *(1.61-3.49)$

$2.58 * * * *(1.62-4.12)$

1.00

3.20 *** (2.05-4.99)

4.II**** $(2.4 I-6.98)$

1.00

$0.5 I^{* * * *}(0.36-0.72)$

$0.23 * * * * 14-0.39)$

1.00

2.02 **** (1.47-2.77)

2.48 **** (1.67-3.70)
1.00

$0.94 \quad(0.69-1.64)$

$0.42 * * *(0.62-1.62)$

$0.79 \quad(0.26-0.75)$

0.33*** (0.43-1.62)

$0.93 \quad(0.20-0.58)$

1.00

1.29 (0.94-1.78)

$0.94(0.62-1.42)$

.00

$2.86 * * *(1.95-4.18)$

$3.75 * * *(2.35-5.99)$

1.00

$0.88 \quad(0.64-1.24)$

$0.54 * *(0.36-0.83)$

.00

1.00

$1.99 * * *(1.48-2.69)$

2.23**** (1.54-3.22)
1.00

$0.96 \quad(0.62-1.46)$

$0.92 \quad(0.57-1.46)$

$0.43 * * *(0.26-0.69)$

0.83 (0.43-1.6I)

$0.34 * * * *(0.21-0.53)$ 


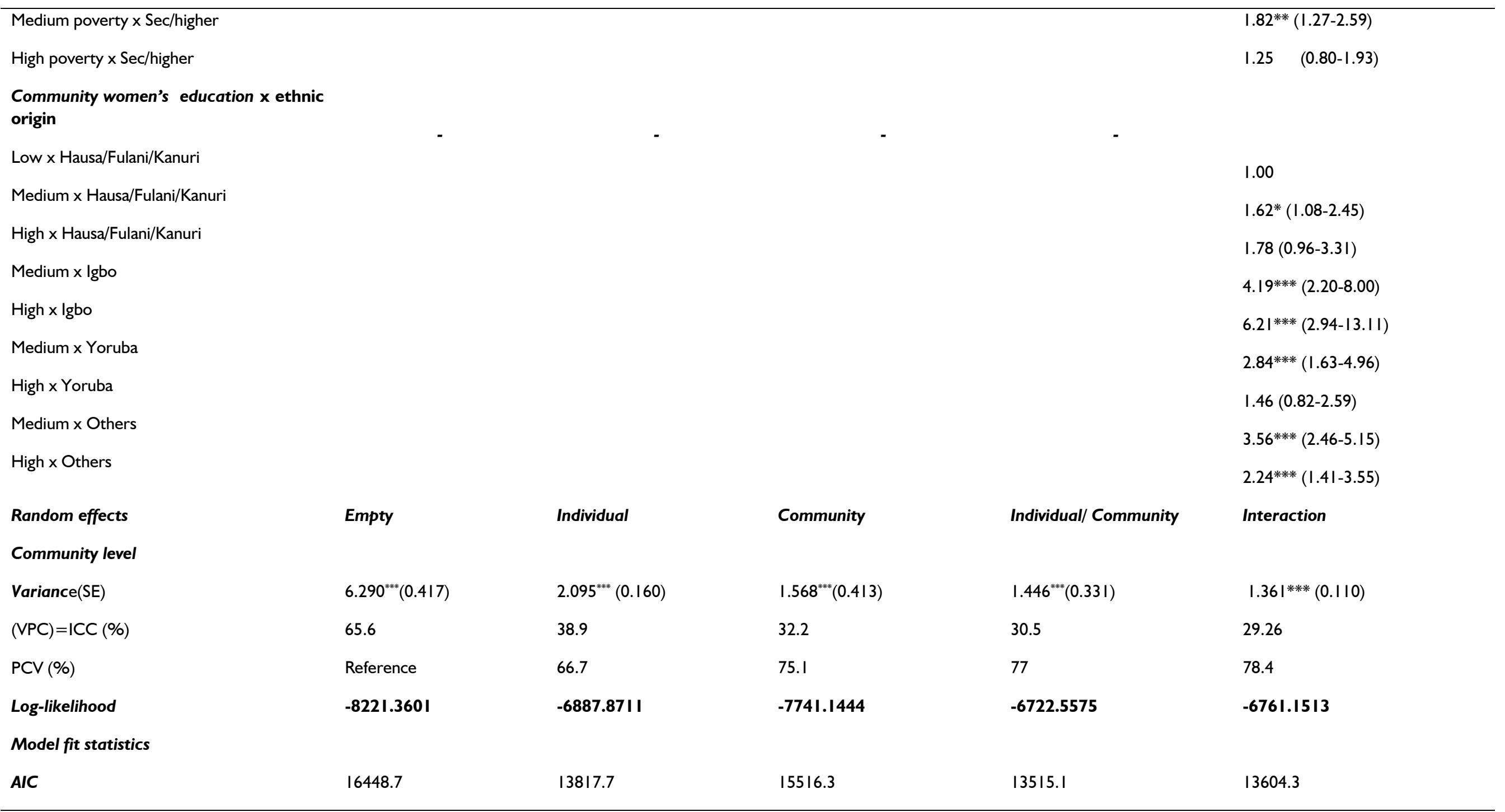

Note: Educational attainment, ethnic origin, community women's education and community poverty were not included in the final model to prevent collinearity. The empty model contains no variables but partitions the variance into two component parts, SE = Standard error, VPC = Variance Partition Coefficient, $\mathrm{PCV}=$ Proportional Change in Variance $\mathrm{AIC}=$ Akaike information criterion, $\mathrm{BIC}=$ Bayesian information criterion

Significance level $* * * \mathrm{p}<0.001 * * \mathrm{p}<0.01 * \mathrm{p}<0.05$ 
$(\mathrm{OR}=0.27, \mathrm{p}<0.00 \mathrm{I})$ and South-East $(\mathrm{OR}=0.46$, $\mathrm{p}<0.00 \mathrm{I})$ was associated with lower odds of using skilled antenatal care compared to living in SouthWest. The odds of receiving skilled ANC was twice higher for women residing in communities with a high proportion of educated women and a high proportion who were exposed to mass media, whereas the odds were 4 times higher for women who lived in communities with a high proportion of health facility delivery. However, women who lived in high poverty communities were less likely to use skilled ANC compared to those who lived in low poverty communities.

In comparison to model I the community variance remained significant $(\tau=1.568, p=0.00 \mathrm{I})$. The intra-community correlation decreased further to $32.2 \%$. The proportional change in variance was estimated at $75 \%$. This shows that including community level factors in this model helped to reduce the community variance from 2.095 ( $\mathrm{p}=$ $0.00 \mathrm{I})$ to I.568 $(\mathrm{p}=0.00 \mathrm{I})$.

Both the individual-level and community-level characteristics were included in model 3 which is the full model. The individual-level factors such as maternal educational attainment, religion, occupation, ethnic origin, a woman's autonomy and household wealth index were significantly associated with the use of skilled ANC. However, result indicates a slight reduction in the odds of skilled ANC utilization across almost all variable categories at both individual and community levels. Similarly, community-level characteristics such as region of residence, community hospital delivery $(\mathrm{OR}=3.75, \mathrm{p}<0.00 \mathrm{I})$, community poverty $(O R=0.54, \mathrm{p}<0.0 \mathrm{I})$ and community mass media exposure $(\mathrm{OR}=2.23$, $\mathrm{p}<0.00 \mathrm{I})$ were significantly associated with skilled ANC.

When compared to model 2 , the variation in ANC across communities was significant $(\tau=$ I.446; $\mathrm{p}<0.00 \mathrm{I})$. The intra-community correlation was $30.5 \%$, while the proportional change in variance was estimated at $77 \%$. The inclusion of the individual and community-level factors reduced the community variance from $1.568(\mathrm{p}=0.00 \mathrm{I})$ to $1.446(\mathrm{p}=$ $0.00 \mathrm{I}$ ) indicating that both individual and community factors were important determinants of the use of skilled ANC.

As shown by the cross level interaction term in Table 2 (Model 4 ) among women who had secondary/higher education, the use of skilled antenatal care significantly increased by residence in medium poverty communities $(\mathrm{OR}=1.82,95 \% \mathrm{Cl}$ : I.27-2.59, $\mathrm{p}<0.0 \mathrm{I})$. For Hausa/Fulani/Kanuri $(\mathrm{OR}=$ I.62, $\mathrm{Cl}=1.08-2.45, \mathrm{p}<0.05)$ and Yoruba $(\mathrm{OR}=$ 2.84, Cl: $(\mathrm{I} .63-4.96, \mathrm{p}<0.00 \mathrm{I})$ women, residence in communities with a medium proportion of educated women increased the odds of receiving skilled ANC. In addition, Igbo women (OR=6.2I, Cl: 2.94-I3.I I, $\mathrm{P}<00 . \mathrm{I})$ and women from other minority ethnic groups $(\mathrm{OR}=2.24, \mathrm{Cl}$ : $(\mathrm{I} .4 \mathrm{I}-3.55, \mathrm{p}<0.00 \mathrm{I})$ who resided in communities with a high proportion of women who had secondary and higher education had higher odds of using ANC from a skilled health provider.

The lower values of AIC in the model indicate a significant improvement of each of the models over previous model.

\section{Discussion}

This study has clearly demonstrated that the inclusion of community level variables was important in explaining the variations in the use of skilled antenatal care. Community-level variables such as region of residence, community hospital delivery, community poverty, and community mass media exposure had strong independent effects on the use of skilled antenatal care. The use of skilled ANC varied across regions. The odds of ANC utilization was higher in South West compared to other regions of Nigeria. The higher likelihood of ANC utilization found among women residing in South Western region is consistent with other studies in Nigeria (Adamu, 20II; Doctor, 20II; NPC \& ICF Macro, 2009). The finding demonstrates that in Nigeria, the characteristics of the region in which women reside influence their utilization of maternal health care services; and suggests differences in socioeconomic development. Furthermore, the result points to the need for regional specific interventions to address the disparities in maternal health care. A better targeting of health policy and planning to enable equitable distribution of need-based resources across the various regions of the country will help to improve the health care system and other infrastructures, which in turn will increase women's access to and demand for maternal health care services.

The study provides evidence that living in communities with a high proportion of women who delivered in a health facility increased the odds of skilled ANC utilization. The finding re-iterates the point that a woman's decisions to use maternal health care may be influenced by availability of health facility and quality of health care in the community (Antai, 2009; Stephenson et al 2006). Living in communities with a high proportion of women from poor households decreased the odds of skilled ANC utilization. This finding lends support to a previous study on reproductive health behaviour (Uthman, 2010). Poverty is a condition of not having enough income to meet basic needs such as food, clothing 
and shelter ( $\mathrm{Vu}, 2005)$. Women, particularly the unemployed are often dependent on their husbands or other family members and are more likely to experience poor maternal health outcomes. From policy standpoint, women from poor households should be empowered economically. Programs to address the issue of community poverty should include strategies to promote economic growth in the community, reduce unemployment and removal of other barriers that prevent economically disadvantaged populations from gaining employment (Shah, 1994).

The odds of skilled ANC utilization were higher for women who lived in communities with a high proportion of women who were exposed to mass media. Living among women who are exposed to any form of mass media could lead to greater awareness of maternal health care services. Furthermore, exposure to mass media may influence women's social attitudes and increase their potential to use skilled ANC. This strongly suggests the need for effective media campaigns regarding the importance of health care services.

At the individual level, educational attainment, occupation, ethnic origin, household wealth index, occupation and religion were significantly associated with the use of skilled ANC. These results are consistent with studies elsewhere (Islam \& Odland 20I I; Kistiana, 2009; Dhaher et al. 2008). Educational attainment, occupation and household wealth status may increase a woman's decision-making power within the household (Matsumura \& Gubhaju 200I) and build greater confidence and capability to make decisions concerning her own health (WHO \& UNICEF 2003). Thus socio-economic empowerment of women may have a reinforcing effect on improved utilization of maternal health care (Amin et al. 20I0).

The effect of ethnic origin (cultural variable) may suggest differentials in socio-economic and cultural characteristics of the different ethnic groups which could influence health care behaviour. For instance, women in some cultures may not seek health care services due to cultural requirements of seclusion (Mesko et al. 2003). Thus the Islamic cultural or religious practices of purdah (seclusion) in Northern Nigeria could contribute to the low utilization of skilled ANC found among Hausa women.

The effect of community poverty and its interaction with maternal education indicates that the odds of ANC utilization increased for women with secondary/higher education resident in low and medium poverty communities. Meanwhile, residence in communities with a high proportion of educated women increased the odds of skilled ANC utilization among women from the entire major and minority ethnic groups. Living in low poverty communities and communities with a high proportion of educated women could enhance women's social and economic status which in turn could increase the use of antenatal care.

The significance of the random intercept highlights the presence of variables that could not be controlled in the multilevel analysis, particularly issues surrounding access to health care such as availability of and actual distance to the health facility which have been established in previous studies to be important determinants of maternal health care utilization (Do, 2008; Mistry et al., 2009). Unfortunately, the analysis could not address the effects of these omitted variables because they were not included in the DHS dataset. Hence, there is need for further research to investigate the unmeasured factors that may account for the unexplained community variations in the outcome variable in this study.

The study has some limitations that are worthy of consideration. The use of the DHS primary sampling unit as the community may bias results due to selection effects. Selection bias recognizes the fact that individuals or families have some degree of choice regarding the communities in which they live ( $\mathrm{Vu}, 2005)$. In consideration of this fact, if important unmeasured individual characteristics influence the choice of individuals (women) to live in certain kinds of communities which influence their health care behaviour either positively or negatively, then the observed effects of community level factors in this study may have been biased.

Some important individual and community factors known to influence service utilization were not included in this study due to limitation of the NDHS data. For example, actual distance to health facility and perceptions of the use of health services. The omission of these important confounders may have biased the estimates of the measured variables in this study.

The study is associated with the problem of 'atomistic fallacy'. The community variables were constructed by aggregating the individual level characteristics at the community level, thus making inferences at a higher level based on information from data collected at the individual level (Boco, 2010).

Despite the identified limitations, the study provides empirical evidence that the community is important in the understanding of variations in the utilization of skilled antenatal care. Moreover, the moderating effects of community factors found in this study, was important in the identification of the community conditions necessary for interventions.

\section{Conclusion}

Community characteristics were important in explaining the variations in the use of skilled ANC. More importantly, community factors moderated the 
association between selected individual factors (education and ethnic origin) and the utilization of skilled antenatal care. The results suggest that improvement in the use of skilled ANC requires increased investment in women's secondary education at the community level. Programs that target poverty alleviation and empowerment of women socially and economically should be encouraged, particularly in disadvantaged communities. These programs have the potential to help women secure means of livelihoods and increase their decision making power regarding the use of maternal health care services.

\section{References}

Abedin, S., Islam, R., \& Hossain, T. (2008). Antenatal care during pregnancy: A study on Naogaon district of Bangladesh. The social science, 3(8), 537-541.

Adamu, H. S. (20I I). Utilization of maternal health care services in Nigeria: An analysis of regional differences in the patterns and determinants of maternal health care use. MSc Unpublished, The University of Liverpool.

Amin, R., Shah, N.M., \& Becker, S. (2010). Socioeconomic factors differentiating maternal and child health-seeking behaviour in Bangladesh: a cross sectional analysis. International Journal for Equity in Health. 9, 9.

Antai, D. (2009). Inequitable childhood immunization uptake in Nigeria: a multilevel analysis of individual and contextual determinants. BMC Infectious Diseases, 9, I8I. doi: 10.1 186/147I-2334-9-I81.

Awusi, V.O., Anyanwu, E.B., \& Okeleke, V. (2009). Determinants of antenatal care services utilization in Emevor village, Nigeria. Benin Journal of Postgraduate Medicine, II, 2I-26.

Babalola, S., \& Fatusi, A. (2009). Determinants of use of maternal health services in Nigeria - looking beyond individual and household factors. BMC Pregnancy and Childbirth, 9, 43. doi: doi: I0.I I86/147|-2393-9-43.

Blalock, H. (1984). Contextual-effects models:theoretical and methodological issues. Annual Review of Sociology, 10, 353-372.

Bloom, S.S., Wypij, D., \& Gupta, M.D. (200I). Dimensions of women's autonomy and the influence on maternal health care utilization in a North Indian city. Demography, 33( I), 67-78.

Boco, A. G. (20I0). Individual and Community Level Effects on Child Mortality: An Analysis of 28 Demographic and Health Surveys in Sub-Saharan Africa DHS Working Papers. Calverton, Maryland, USA.

Burgard, S. A., \& Lee-Rife, S. M. (2009). Community characteristics, sexual Initiation, and condom use among young black South Africans. Journal of Health and Social Behavior, 50(3), 293-309.

Dhaher, E., Mikolajczyk, R. T., Annette, E., Maxwell, A. E., \& Krämer, A. (2008). Factors associated with lack of postnatal care among Palestinian women: A cross-sectional study of three clinics in the West Bank. BMC Pregnancy and Childbirth, 8, 26. doi: I0.I I86/I47|I-2393-8-26.

Do, M. (2008). Antenatal care service availability and utilization in rural Viet Nam. Asia-Pacific Population Journal, 23(I), 29-54.

Doctor, H. V. (20I I). Intergenerational differences in antenatal care and supervised deliveries in Nigeria. Health \& place, 17, 480-489.

Dolla, C.N. (2008). Utilization of antenatal care service in Madhya Pradesh India. MSc, Free University, Amsterdam.

Gage, A. J. (2007). Barriers to the utilization of maternal health care in rural Mali. Social Science \& Medicine, 65(8), I666-I682.

Galadanci, H.S., Ejembi, C.L., Iliyasu., Z , Alagh., B, \& Umare, U.S. (2007). Maternal health in Northern Nigeria-a far cry from ideal. An International Journal of Obstetrics and Gynaecology, I I4, 448452. doi: I0. I I I /j. I47I-0528.2007.0I229.x

Griffiths, P., \& Stephenson, R. (200I). Understanding users' perspectives of barriers to maternal health care in Maharashtra, India. Journal of Biosocial Science, 33(3), 339-359.

Halfon, N., Inkelas, M., \& Hochstein, M. (2000). The health development organization: an organizational approachto achieving child health development. Milbank Quaterly, 78, 447-497.

Ikamari, L.D.E. (2004). Maternal health care utilization in Teso district. African Journal of Health Science, II, 2I-32.

Islam, M. R., \& Odland, J. O. (20I I). Determinants of antenatal and postnatal care visits among Indigenous people in Bangladesh: a study of the Mru Community. Rural and Remote Health Journal.Retrieved from http://www.rrh.org.au/articles/subviewasia.asp?Ar ticlelD $=1672$.

Kistiana, S. (2009). Socio-economic and demographic determinants of maternal health care utilization in Indonesia. Msc Thesis. The Flinders University of Australia Adelaide.

Mackian, S. (2003). A review of health seeking behaviour: problems and prospect Working paper. University of Manchester: Manchester.

Magadi, M. A., Zulu, E. M., \& Brockerhoff, M. (2003). The inequality of maternal health care in urban sub-Saharan Africa in the 1990's. Population studies, 57(3), 347-366.

Matthews, S.A., \& Gubhaju, B. (2004). Contextual influences on the use of antenatal care in Nepal 
DHS Geographic Studies 2. Calverton, Maryland USA.

Matsumura, M., \& Gubhaju, B. (200I). Women's status, household structure and the utilization of maternal health service in Nepal. Asia-pacific population journal, I6(I), 23-44.

Mbuagbaw, L.C.E., \& Gofin, R. (20II). A new measurement for optimal antenatal care: Determinants and outcomes in Cameroon. Maternal and Child Health Journal, 5(8), 1427-34. doi: 10. 1007/s 10995-010-0707-3.

Merlo, J., Chaix, B., Ohlsson, H., Beckman, A., Johnell, K., Hjerpe, P., Ra ${ }^{\circ}$ stam, L., \& Larsen, K. (2006). A brief conceptual tutorial of multilevel analysis in social epidemiology: using measures of clustering in multilevel logistic regression to investigate contextual phenomena. Journal of Epidemiology and Community Health, 60, 290297. doi: doi: 10.1 I36/jech.2004.029454.

Mesko, N., Osrin, D., Tamang, S., Shrestha, B. P., Manandhar, D. S., Manandhar, M., \& Costello, A. M. (2003). Care for perinatal illness in rural Nepal: A descriptive study with cross-sectional and qualitative components. BMC International Health Human Rights, 3(I), 3.

Mistry, R, Galal, O, \& Lu, M. (2009). Women's autonomy and pregnancy care in rural India: A contextual analysis. Social Science \& Medicine, 69, 926-933.

National Population Commission (NPC) [Nigeria] \& ICF Macro. (2009). Nigeria Demographic and Health Survey 2008. Abuja, Nigeria: National Population Commission and ICF Macro.

Oguntunde, O., Aina, O., Ibrahim, M.S., Hajara S.Umar., H.S., \& Passano, P. (20I0). Antenatal care and skilled birth attendance in three communities in Kaduna state, Nigeria. African Journal of Reproductive Health I4(3), 89.

Ojanuga, D.N., \& Gilbert. (1992). Women's access to health care in developing countries. Social Science and Medicine, 35(4), 613-617.

Onah, H. E., Ikeako, L. C., \& Iloabachine, G. C. (2006.). Factors associated with the use of maternity service in Enugu south eastern Nigeria. Social science and medicine, 63, 1870-1878.

Ononokpono, D. N., Odimegwu, C. O., Imasiku, E., \& Adedini, S. (20I3). Contextual determinants of maternal health care service utilization in Nigeria. Women \& Health, 53(7), 647-668.

Rahman, S. A. (2000). Utilization of Primary Health Care Services in Rural Bangladesh: The population and provider perspectives. PhD. thesis. University of London, London

Robert, S., \& House, J. (2000). Socioeconomic inequalities in health: integrating individual, community and social level theory and research. In Scrimshaw S Fitzgerald RM (ed.), The handbook of social studies in health and medicine (pp. II5- I35). London: Sage.

Rutstein, S. O., \& Johnson, K. (2004). The DHS Wealth Index. The DHS comparative report. Calverton, Maryland USA.

Shah, C.P. (1994). Public health and preventive medicine in Canada (3rd ed.). Toronto: University of Toronto Press.

Shaikh, B. T., Haran, D., \& Hatcher, J. (2008). Women's social position and health-seeking behaviours: is the health care system accessible and responsive in Pakistan? Health care for women international, 29 (8), 945-956. doi: I0.1080/07399330802380506.

Shaikh, B.T., Haran D., \& Hatcher, J. (2004). Where do they go, whom do they consult, and why? Health-seeking behaviours in the northern areas of Pakistan. Qualitative Health Research, 18:747. doi: I0. I I77/I0497323083 I 7220.

Simkhada, B., Porter, M. A., \& van Teijlingen, E. R. (20I0). The role of mothers-in-law in antenatal care decision-making in Nepal: a qualitative study. BMC Pregnancy and Childbirth I0, 34.

Stephenson, R., Baschieri, A., Clements, S., Hennink, M., \& Madise, N. (2006). Contextual Influences on the Use of Health Facilities for Childbirth in Africa. American Journal of Public Health, 96(I), 84-93. doi: I0.2 I 05/ajph.2004.057422

Stokols, D. (1996). Translating social ecological theory into guidelines for community health promotion. American Journal of Health Promotion, I0(4), 282-298.

Titaley, C. R., Hunter, C. L., Heywood, P., \& Dibley, M. J. (20I0). Why don't some women attend antenatal and postnatal care services?: a qualitative study of community members' perspectives in Garut, Sukabumi and Ciamis districts of west Java province Indonesia. BMC Pregnancy and Childbirth. 10,61.

Tiwari, D. (20/0). Utilization of maternal health services in Uttar Pradesh: a comparison between simple logistic and multilevel logistic regression analysis. Paper presented at the Population Association of America, Dallas, United States of America.

Trinh, L.T.T., Dibley, M.J., \& Byles, J (2007). Determinants of antenatal care utilization in three rural areas of Vietnam. Public Health Nursing, 24(4), 300-310.

Uthman, O. A. (2010). Does It Really Matter Where You Live? A Multilevel Analysis of Social Disorganization and Risky Sexual Behaviours in Sub-Saharan Africa DHS Working paper. Calverton, Maryland, USA.

$\mathrm{Vu}$, L. T. H. (2005). Multilevel determinants of children's health outcomes. PhD. Thesis University of Saskatchewan, Canada. 
Wall, L. L. (1998). Dead mothers and injured wives: the social context of maternal morbidity and mortality among the Hausa of northern Nigeria. Studies in Family Planning, 29(4), 34I-359.

World Bank (2014). Trends in maternal mortality: 1990-2013. Estimates developed by WHO, UNICEF, UNFPA and The World Bank and United Nations Population Division Geneva.
World Health Organization (WHO) (20I4). Trends in maternal mortality: 1990 to 2013 Estimates by WHO, UNICEF, UNFPA, The World Bank and United Nations Population Division. Geneva, Switzerland.

World Health Organization \& UNICEF. (2003). Antenatal Care in Developing Countries: Promises, Achievements and Missed Opportunities: An Analysis of Trends, Levels, and Differentials: 1990-200I. Geneva. 